\title{
A rare coronary anomaly: all main coronary arteries originating from right sinus of Valsalva with separate ostium
}

\author{
Erdal Gursul', Birsel Sen Akova ${ }^{2}$, Ali Ozturk $^{3}$ \\ 'Biga State Hospital, Department of Cardiology, Canakkale, Turkey \\ ${ }^{2}$ Biga State Hospital, Department of Radiology, Canakkale, Turkey \\ ${ }^{3}$ Sifa University, Department of Cardiology, Izmir, Turkey
}

\begin{abstract}
All coronary arteries originating from separate ostia in the right sinus of Valsalva is a very rare coronary artery anomaly. In particular, the inter-arterial course of the coronary arteries may lead to malignant clinical outcomes. In the literature, such coronary artery anomaly have been reported to cause myocardial ischaemia and sudden cardiac death. In our case, coronary angiogram was performed on a patient presenting with unstable angina pectoris, and the left anterior descending artery, circumflex artery and right coronary artery were found to originate from separate ostia in the aorta. To better clarify the course of the coronary arteries, coronary computed tomography angiography was performed and all three coronary arteries were determined to originate from separate ostia in the right sinus of Valsalva. The left anterior descending artery was found to pass through the inter-atrial septum in coronary computed tomography angiography. In our presentation, the clinical data about the case where three coronary arteries originated from separate ostia in the right sinus of Valsalva have been presented along with the coronary angiography and coronary computed tomography angiography images in the light of literature.
\end{abstract}

Key words: coronary artery anomaly, coronary computed tomography angiography, coronary angiography

Acta Angiol 2015; 21, 2: 57-60

\section{Introduction}

Coronary artery anomalies (CAAs) are disorders which are rare, but may lead to angina and sudden cardiac death. Since CAAs are often asymptomatic, the precise incidence data are not available. The frequency has been reported as $0.3-0.78 \%$ in autopsy series and $1-1.3 \%$ in angiogram series [I-4].

Most of the CAAs are outlet and distribution anomalies [4]. The outlet of left coronary system from the right sinus of Valsalva (RSV) may have a malignant progression in CAA. Notably, the pressure on the coronary arteries while passing between the pulmonary artery and the aorta in an abnormal course may cause arrhythmia, angina, myocardial ischaemia, and even sudden cardiac death $[5,6]$.

The outlet of left anterior descending artery (LAD), circumflex artery $(C x)$ and right coronary artery (RCA) from RSV using separate ostia, without left main coronary artery is a very rare CAA [7]. In our case, the conventional angiogram and coronary computed tomography angiography (CCTA) images belonging to a patient who had presented to the emergency department with angi- 
na symptoms and where coronary angiography (CAG) revealed a rare CAA with $L A D, C x$ and RCA originating from separate ostia in RSV have been presented.

\section{Case study}

A 65-year-old woman was admitted to the emergency department with typical anginal chest pain of 3 hours duration. Any previous chronic disorder was not present as well as a family history of coronary artery disease of early age. Systolic/diastolic blood pressure was 123/ $/ 75 \mathrm{~mm} \mathrm{Hg}$ and pulse was 96/min during admission. No significant pathology was found on the cardiac evaluation. On the electrocardiogram (ECG), sinus rhythm was observed and no ischaemic sign was seen. At first, the troponin value was within normal limits; the patient was monitored and a repeat troponin test was planned after 4 hours. The control troponin value was also normal and no ischaemic changes were observed on the ECG. As the typical anginal chest pain recurred on the follow up, CAG was planned with the pre-diagnosis of unstable angina pectoris. CAG was performed utilizing the transfemoral approach and the Judkins technique. Since the left system was not visualized with the 5-F Judkins catheter, RCA cannulation was performed first. RCA (dominant artery) was visualized with the Judkins right $4 \mathrm{~F}$ catheter and posterior descending artery was found to extend to the apex (Fig. IA). Cx cannulation was performed with the same catheter and the $C x$ was long and twisted till the obtuse marginal branch (Figs. IB and 2A). LAD could be visualized using a multi-purpose catheter. The LAD course was found to be narrow and abnormal (Fig. 2B). According to CAG, the LAD, Cx and RCA originated from separate ostia in the aorta (Figs. I and 2). No luminal narrowing or plaque formation was observed in the coronary arteries. The patient was diagnosed as having coronary artery outlet anomaly, and to clarify the coronary artery course, a CCTA was planned. CCTA was performed with 64-slice computed tomography (Toshiba Aqullion 64, Toshiba Medical Systems, Tokyo, Japan). The imaging protocol
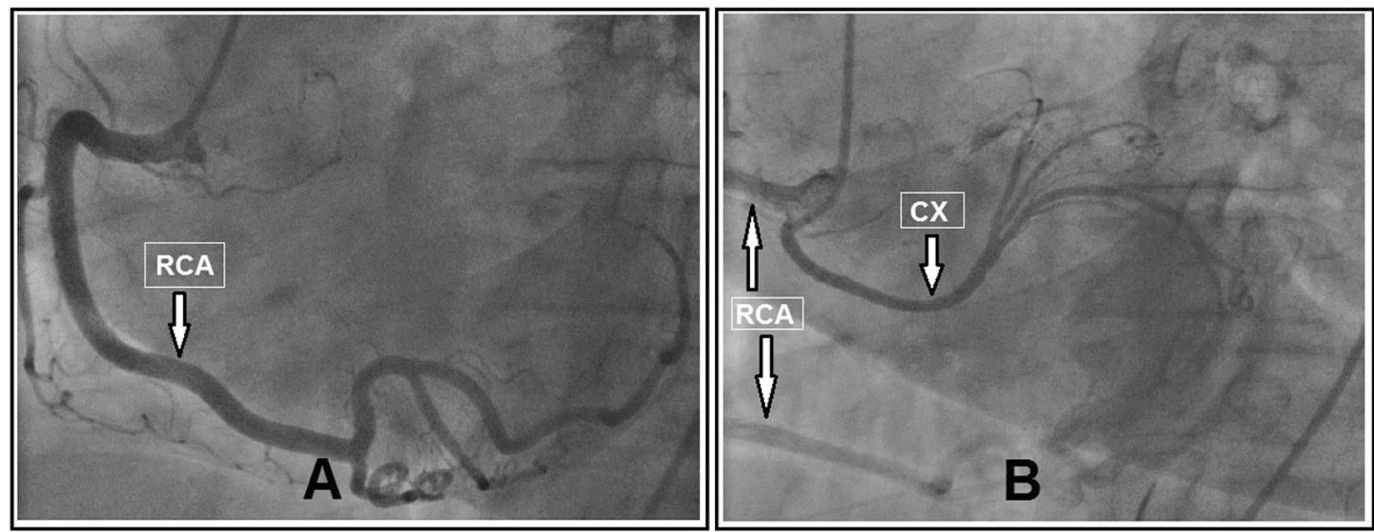

Figure I. Coronary angiography images of right coronary artery (RCA) and circumflex artery (Cx). A. RCA which was the dominant artery was observed in the routine course. B. Cx was cannulated near the RCA ostium at the right sinus of Valsalva
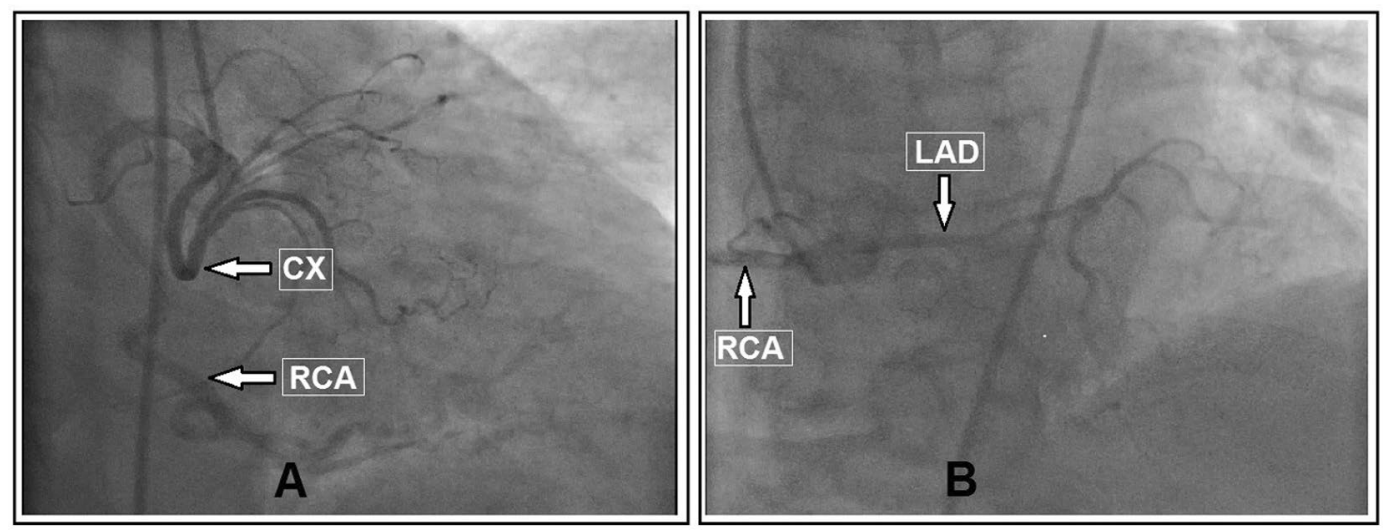

Figure 2. Coronary angiography images of circumflex artery $(C x)$ and left anterior descending artery (LAD). A. Cx was observed to be tortuous during the long course. B. LAD that could not be precisely cannulated was seen to be narrow 


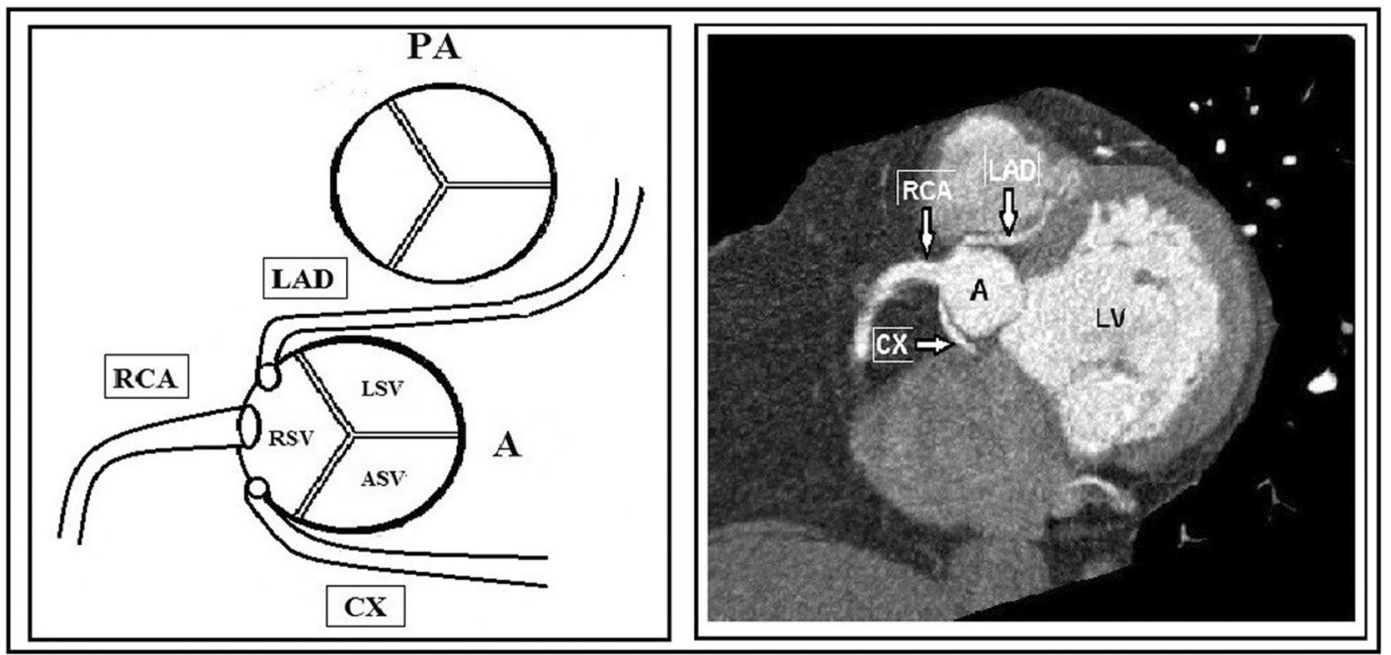

Figure 3. Coronary computed tomography angiogram images of the aorta. The left anterior descending artery, circumflex artery and right coronary artery originated from separate ostia in the aorta. A — aorta, PA — pulmonary artery, LAD — left anterior descending artery, Cx — circumflex artery, RCA — right coronary artery, RSV — right sinus of Valsalva, LSV — left sinus of Valsalva, ASV - aortic sinus of Valsalva, LV — left ventricle

included $120 \mathrm{kV}, 400 \mathrm{mAs}, 0,5 \mathrm{~mm}$ slice thickness, $400 \mathrm{~ms}$ gantry rotation rate and $200 \mathrm{~mm}$ field of view using ECG gating. Vascular access was from the right antecubital vein with $20 \mathrm{G}$ catheter. After administering $350 \mathrm{mg} / \mathrm{ml}$, $80 \mathrm{ml}$ non-ionic contrast material at $5 \mathrm{ml} / \mathrm{sec}, 20 \mathrm{ml}$ saline infusion was given at the same rate. The automatic bolus triggering method was used to determine the contrast material delay time. Images were reconstructed in $75 \%$ $\mathrm{R}-\mathrm{R}$ interval. 2 two-dimensional and three-dimensional images were obtained from the reconstructed images by post-processing at the work station (Vitrea 2, vital image INC, Plymouth, Minnesota, USA). On the CCTA, the LAD, $C x$ and RCA were observed to originate from separate ostia in the right coronary sinus (Fig. 3). The dominant artery, RCA, was in a normal course and the posterior descending artery extended until the apex. The $C x$ was narrow and passing to the posterior of the aorta, and ended after giving obtuse marginal branches. The LAD first passed to the anterior of the aorta and then, between the pulmonary artery and the aorta. During this passage, it was narrow and when the passage ended, it became dilated and extended to the apex. The patient was informed about CAA, the risks were described and treatment alterations were discussed. $\beta$-blocker therapy was prescribed to the patient who refused invasive procedures.

\section{Discussion}

CAAs that mostly run an asymptomatic course may gain a malignant character in cases when a coronary artery originates from the opposite aortic sinus. The CAG incidence is $0.017 \%$ where the left coronary artery originates from the RSV, and its incidence in CAA is $1.3 \%[4,8]$. As the left coronary system may originate from the same ostium with right coronary artery, all three coronary arteries may also originate from separate ostia in RSV. In the literature, CAA cases where 3 coronary arteries originated from separate ostia in RSV were seen in 7 patients in the study of Kashyap et al., in 3 patients in the study of Clik et al., in I patient in the study of Engel et al., and in I patient in the study of Yamanaka and Hobbs [I, 4, 9, I0]. Al-Mohaissen et al. reviewed 34 cases from the literature and revealed that RCA often had a normal course, Cx mostly ran behind the aortic root and rarely in front of the pulmonary artery or at the interventricular septum; the LAD mostly ran in front of the pulmonary artery, septum or the interatrial septum [II].

Outlet of the right and left coronary system from RSV is an uncommon CAA, but it may be symptomatic. There are articles on cases with no atherosclerosis, but presenting with myocardial ischaemia and sudden cardiac death following exercise $[5,6,12]$. This has been attributed to blood volume decrease and even to total coronary artery occlusion, when there is pressure on the left main coronary artery and/or its branches during their passage through the aorta and pulmonary arteries, due to the rise in volume of these arteries during exercise $[5,6]$. Some authors thought that myocardial ischaemia occurred due to a vasospasm that may have developed as a result of endothelial damage due to an abnormal course [12]. Since there was no critical narrowing, typical anginal pain in our case may have been related to the reasons discussed above. 
The treatment alternatives for CAA are still controversial. Treatment must be individually discussed. Revascularization is recommended, mainly for CAA patients below 35 years carrying the risk of sudden cardiac death [12-14]. Some articles state that $\beta$-blocker may have benefit in CAA patients who refuse revascularization [15-17]. In our case, the probable risks and treatment alternatives were described and then, $\beta$-blocker therapy was prescribed since our patient refused invasive procedures.

In this report, the CAG and CCTA images that have been presented belong to a case in which the LAD, Cx and RCA originate from separate ostia in RSV and where LAD has been investigated, since it was probably subject to pressure while running between pulmonary artery and aorta.

\section{Conclusion}

CAA in which the LAD, Cx and RCA originate from separate ostia in RSV is a very unique anomaly, but has a malignant potential such as sudden cardiac death. Advanced evaluations such as CCTA are valuable and complementary to CAG for definition of the origin and course of coronary arteries, principally in anomalies that may have a malignant character.

\section{References}

I. Patel KB, Gupta H, Nath $\mathrm{H}$ et al (2007) Origin of all three major coronary arteries from the right sinus of Valsalva: clinical, angiographic, and magnetic resonance imaging findings and incidence in a selectreferral population. Catheter Cardiovasc Int; 69: 7I I-7I8.

2. Larsen Al, Ørn S, Barvik S, Nilsen DW (2007) Anomalies of the coronary arteries originating from the right sinus of Valsalva. (I) Single coronary artery originating from the right sinus associated with fusion of the left and the non coronary cusp and atrophy of the left coronary ostium. (2) Three separate coronary arteries originating from the right sinus of Valsalva. Int J Cardiol; I I5: 86-89.

3. Zhang F, Ge JB, Qian JY, Fan B, Wang QB, Chen HZ (2005) Frequency of the anomalous coronary origin in the Chinese population with coronary artery stenosis. Zhonghua Nei Ke Za Zhi [Chinese Journal of Internal Medicine]; 44: 347-349.
4. Yamanaka O, Hobbs RE (1990) Coronary artery anomalies in 126,595 patients undergoing coronary arteriography. Catheter Cardiovasc Diagn; 21: 28-40.

5. Cheitlin MD, DeCastro CM, McAllister A (1974) Sudden death as a complication of anomalous left coronary origin from the anterior sinus of Valsalva A not-so-minor congenital anomaly. Circulation; 50: 780-787.

6. Salloum JA, Thomas D, Grosgogeat Y (1992) Anomalies de naissance des coronaires: responsabilité dans l'ischémie myocardique. La Presse médicale; 21: I I72-I I 74.

7. Bartorelli AL, Capacchione V, Ravagnani P, Pepi M (1994) Anomalous origin of the left anterior descending and circumflex coronary arteries by two separate ostia from the right sinus of Valsalva. Int J Cardiol; 44: 294-298.

8. Chaitman BR, Lesperance J, Saltiel J, Bourassa MG (1976) Clinical, angiographic, and hemodynamic findings in patients with anomalous origin of the coronary arteries. Circulation; 53: |22-|3|.

9. Click RL, Holmes DR, Vlietstra RE, Kosinski AS, Kronmal RA (1989) Anomalous coronary arteries: Location, degree of atherosclerosis and effect on survival. A report from the Coronary Artery Surgery Study. J Am Coll Cardiol; 13: 53 I-537.

10. Engel HJ, Torres C, Page HL (1975) Major variations in anatomical origin of the coronary arteries: Angiographic observations in 4,250 patients without associated congenital heart disease. Catheter Cardiovasc Diagn; I: 157-169.

II. Al-Mohaissen M, Heilbron B, Leipsic J, Ignaszewski A (2010) Anomalous origin of the entire coronary system by three separate ostia within the right coronary sinus - a rarely observed coronary anomaly. Canad J Cardiol; 26: 206-208.

12. Basso C, Corrado D, Thiene G (200I) Congenital coronary anomalies as an important cause of sudden death in the young. Cardiol Rev; 9: 312-317.

13. Pellicia A (200I) Congenital coronary artery anomalies in young patients. New perspectives for timely identification. J Am Coll Cardiol; 37: 598-600.

14. Taylor AJ, Virmani R (200I) Coronary artery anomalies in adults: which are high risk? ACC Curr J Rev; 10: 92-95.

15. Kaku B, Shimizu M, Yoshio H et al (1996) Clinical features on prognosis of Japanese patients with anomalous origin of the coronary artery. Jap Circulation J; 60: 73I-74I.

16. Abouzied AM, Amaram S, Neerukonda SK (1999) Anomalous left coronary artery arising from right sinus of Valsalva could be a minor congenital anomaly. A case report and review of the literature. Angiology; 50: 175-178.

17. Bixby MB (1988) Successful medical management of a patient with an anomalous right coronary artery who declined surgery. Am J Crit Care; 7: 393-394. 